\title{
Advancing Mindful Parenting Research: an Introduction
}

\author{
Justin Parent $^{1}$ (D) $\cdot$ Karissa DiMarzio ${ }^{1}$ \\ Accepted: 28 November 2020 / Published online: 6 January 2021 \\ (C) The Author(s), under exclusive licence to Springer Science+Business Media, LLC part of Springer Nature 2021
}

Mindful parenting is not a new concept to the literature. In fact, as Anālayo (2021)) has illustrated in his paper, principles of mindful parenting can be seen as early as the Pāli Canon, with many parallels observed between the Buddha's teachings and the modern pioneering work of Kabat-Zinn and KabatZinn (1997). With substantial evidence supporting the connection between mindfulness practices and improved health outcomes, it was inevitable that there would be empirical shifts towards how these practices could be cultivated and applied to everyday family life. Indeed, we have seen a rapid increase in mindful parenting empirical research since Dumas' (2005) seminal work calling for increased attention to how mindfulness can enhance the family system and intervention program (see Fig. 1). In particular, over the last two decades, mindful parenting has emerged as a promising mechanism of change in families impacted by various intra- and interpersonal challenges, including elevated parenting stress (e.g., Bögels et al. 2014; Chaplin et al. 2021), youth behavioral difficulties (e.g., Brown et al. 2021; Potharst et al. 2021a; Singh et al. 2021), and coparenting dysfunction (e.g., Bögels et al. 2014). Despite increasing attention, however, systematic reviews have highlighted the need for increased methodological rigor, citing small sample sizes, lack of randomization, and single-informant reporting as barriers to mindful parenting becoming a well-established contributor to parent and youth mental well-being (e.g., Burgdorf et al. 2019; Townshend et al. 2016). While such limitations are due, at least in part, to the comparatively nascent status of this work, these critiques have been helpful in shedding light on needed areas for improvement that are imperative to the advancement of mindful parenting research.

Justin Parent

jparent@fiu.edu

1 Department of Psychology, Center for Children and Families, Florida International University, Miami, FL, USA
The purpose of this Special Issue is to highlight the new and innovative research being conducted in this area and to bring attention to the clinically meaningful and wide-reaching implications of mindful parenting as a target for intervention. Within this issue, readers will find a diverse range of papers, from exploratory correlational analyses to evaluations of clinical trials across various populations and settings. Although each paper is unique in its approach and overarching goal, the collection as a whole underscores the appeal of disseminating mindful parenting interventions on a broader scale, particularly given the promising effects observed in at-risk families as well as the relatively limited resources needed for implementation.

To begin, the Special Issue opens with a commentary from Kabat-Zinn and Kabat-Zinn (2021), providing reflections on their original definition of mindful parenting, how it has evolved over the past two decades, its importance at this time of stress and disruption, and insights into future directions. Following this commentary and the one referenced above by Anālayo (2021), the issue begins its empirical coverage with six correlational papers exploring associations between parental factors, mindful parenting, and child psychosocial health. Fernandes et al. (2021a) have contributed two papers from their work in Portugal, the first of which examines new mothers and their children postpartuma transitional and often stressful period marked as a cornerstone to the development of the subsequent parent-child relationship. The authors found that mothers who perceived their child as having a difficult temperament reported higher levels of stress and fewer mindful parenting practices, highlighting parenting stress as a noteworthy mechanism with important implications for new mothers' postpartum adjustment. Using a similar sample, Fernandes et al. (2021b) complemented this work with their examination of maternal preferences and acceptability of mindful parenting interventions. In this paper, $95 \%$ of mothers reported feeling that such interventions would be useful to them during the postpartum period. Mothers also reported a preference for an average of 10 weekly sessions each ranging from 45 to $60 \mathrm{~min}$, lending insight not only to the acceptability of these interventions but also to the aspects that may be critical to their feasibility in community settings. 
Fig. 1 Growth in mindful parenting research

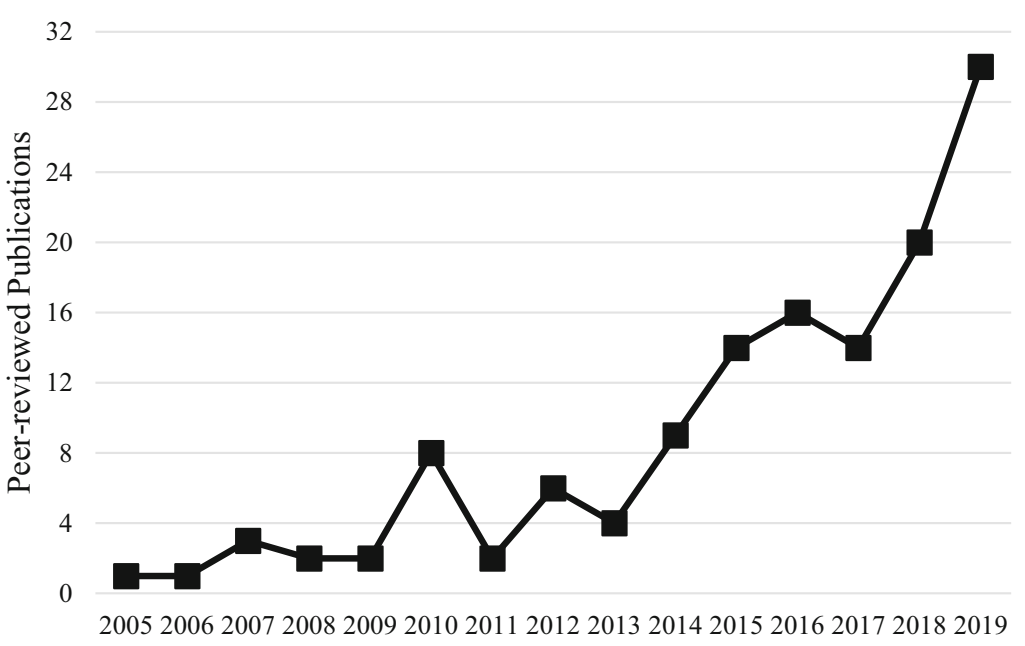

Relatedly, Ren et al. (2021) examined parenting stress in an Eastern sample of Chinese parents, but in the context of mothers' use of parenting practices and children's emotion regulation. Their results also suggested the importance of targeting parenting stress given its potential to undermine the benefits of mindfulness otherwise observed in the literature. In a different vein, Moreira et al. (2021) explored mindful parenting as an underlying mechanism of the relationship between parent/child negative affect and use of overprotective and supportive parenting practices. Their work draws attention to the affective tendencies of parents and their children, and the significant correlations observed between both and multiple facets of mindful parenting (i.e., parents' ability to listen with full attention, emotional awareness of and compassion for the child). In addition, Han et al. (2021) explored correlations between Chinese parents' dispositional mindfulness, use of mindful parenting practices, and children's psychopathology. Despite illuminating ways in which these associations may differ between Western and Eastern samples, the findings suggest that mindful parenting (or lack thereof) might play just as influential a role in the development of psychopathology among Chinese youth as observed in their Western counterparts. Lastly, Potharst et al. (2021a, b, c) examined whether maternal reports on the Interpersonal Mindfulness in Parenting Scale (IMP-S; Duncan 2007) predict actual engagement in mindful parenting practices during an observational task with children. Their work illuminates which facets of the IMP-S are likely to be more accurate predictors of maternal behaviors (i.e., positive affect, childcentered attention) and cautions researchers from discounting the value of subscale scores when assessing the quality of parent-child interactions.

From here, we transition to the five longitudinal papers that were selected for inclusion in the Special Issue. The first three evaluate the mediational role of mindful parenting, beginning with Parent et al.'s (2021) examination of how parent dispositional mindfulness contributes to children's internalizing and externalizing symptomology. Findings showcase how higher levels of parent mindfulness predict greater use of mindful parenting strategies, which in turn influences parents' positive and negative parenting and consequently youth mental health outcomes. Importantly, the authors highlighted the robust impact of mindfulness on youth outcomes across early childhood and adolescence, suggesting a potentially ubiquitous benefit to increasing parents' mindfulness regardless of child age. Next, Henrichs et al. (2021) examined whether mindful parenting mediates the relationship between maternal anxiety and child psychosocial problems. Findings suggest that maternal anxiety in pregnancy can persist into early childhood and impact parents' engagement in mindful parenting. This cascade, in turn, was found to increase children's likelihood of exhibiting internalizing behaviors, positing pregnancy as a potential period for early prevention and intervention services. A work contributed by Lippold et al. (2021) suggests that parental cognitions may also be an important target for improvement, particularly those related to parents' sense of competence and self-attributions related to parenting. These authors drew attention to the relationship between mindful parenting and positive parenting cognitions, and how both factors might influence and perpetuate each other over time. They also noted that mindful parenting appeared to mediate the relationship between positive parenting cognitions and adaptive parent-child communication, which might further clarify how mindful parenting promotes positive youth outcomes. Overall, findings from these three papers not only present potential targets for prevention and intervention services but also showcase the long-term impact of enhanced mindful parenting on family functioning.

The remaining two prospective investigations included in the current issue contribute to the literature on the evaluation and effectiveness of mindful parenting interventions. Turpyn et al. (2021) explored the impact of a mindful parenting intervention on maternal brain functioning and connectivity. The 
authors found several notable alterations to brain regions, such as the posterior insula and dorsal anterior cingulate cortex, which were associated with mothers' increased engagement in mindful parenting. Their study marks the first evaluation of the neurobiological mechanisms of a mindfulness-based parenting intervention and helps strengthen our confidence in the effectiveness of these approaches. Lastly, Calvete et al. (2021) not only provided further support for the positive association between mindful parenting and children's well-being but also lend novel contributions to the literature through their examination of children's dispositional mindfulness as a moderator of this relationship. In particular, the authors found that parents' use of mindful parenting practices may be more beneficial to children who have poorer dispositional mindfulness. Together, these investigations add to our understanding not only of how mindful parenting interventions might work but also for whom such interventions might work best.

The remaining nine papers in the Special Issue focus more exclusively on evaluations of mindful parenting programs. A majority of the papers demonstrate reductions in parenting stress, and more than half evaluate the impact of these programs in at-risk samples, ranging from parents with clinically elevated psychopathology to those with histories of economic or relational adversity. The section begins with Brown et al.'s (2021) examination of child welfare-involved families. Compared to those randomized to the waitlist, families who received the intervention reported improvements in both proximal and distal domains of functioning, including the risk of child maltreatment, child behavior problems, and both selfreport and physiological indices of parental stress. While the program was not shown to have an impact on parents' use of substances as a coping strategy, Brown et al. did demonstrate the feasibility of implementing such a program in the child welfare system. This is noteworthy as the integration of child protective services and mindful parenting programs could have implications for the long-term outcomes of these families, given their risk of repeated referrals (e.g., Casanueva et al. 2015; Hélie et al. 2014) as well as the detrimental impact these referrals have on children's psychosocial well-being ( $\mathrm{Li}$ and Godinet 2014).

In a similar vein, Lengua et al. (2021) examined the feasibility of integrating a brief parenting intervention into existing early learning programs that support children from economically disadvantaged backgrounds. The program combined traditional behavioral parent training with components meant to promote parents' own sense of well-being, including their engagement in mindfulness and emotion regulation practices. Overall, their evaluation provides preliminary support for the program's feasibility within these settings and also for its effectiveness in improving both parent and child self-regulation and adaptive functioning. The authors' focus on economically disadvantaged families is important given the higher rates of stress reported by parents of low socioeconomic status. In their paper, Chaplin et al. (2021) provided a comprehensive overview of the implications of elevated parenting stress and the impact that stress can have on the parent-child relationship. This association is, in part, why many mindfulness programs, including a majority of those discussed in the current issue, target parenting stress as a mechanism of change in the family system. The support provided by these first two studies is important given they demonstrate two methods of providing early intervention services to at-risk parents - services that are not only acceptable to target families but also feasible for the involved agencies. The latter of which is of particular importance given that such agencies are often overburdened as a result of operating in lower resource settings, where feasibility is integral to the successful dissemination of services. Relatedly, the work contributed by Chaplin et al. serves as yet another excellent demonstration of how mindfulness skills can be used as a tool for reducing parenting stress and improving parent-child relationship quality. The authors also noted important moderation effects in that improvements in relationship quality appear more pronounced for mother-daughter dyads after participation in a mindfulness-based intervention than for mother-son dyads.

Singh et al. (2021) and Lönnberg et al. (2021) also evaluated families at risk of experiencing elevated parenting stress. Singh et al. evaluated the efficacy of a Mindfulness-Based Positive Behavior Support (MBPBS) program for mothers of children diagnosed with autism spectrum disorder. Compared to those in the component groups (i.e., MB only and PBS only), mothers in the combined MBPBS experienced greater reductions of stress and children's aggressive behaviors. Importantly, while the MB group experienced greater improvements than the PBS group, neither was superior to the combined MBPBS program, demonstrating the optimum of addressing parental well-being in addition to child behavioral management. In addition, Lönnberg et al. (2021) evaluated the efficacy of a mindfulness-based intervention for women pregnant with their first child and at risk for perinatal depression. They, too, observed significant improvements among mothers in the intervention group, including reductions in self-reported stress and depressive symptomatology, as compared to those in the control group. Unlike Singh et al., the authors did not observe sustained effects at the follow-up assessment; however, they did observe that women who maintained their mindfulness practice sustained effects more so than those who discontinued their practice. This in conjunction with Singh et al.'s findings on the predictive power of total meditation time per day underscore the necessity of maintained practice - an aspect that may require effortful transparency with parents at the onset to ensure they understand that mindfulness, like any other skill, requires honing through ongoing practice.

Potharst contributed two additional first-authored papers to the Special Issue. The first of these papers, Potharst et al. 
(2021a) examined a group training for clinically referred mother-toddler dyads. Like the previous papers, the authors observed significant improvements across multiple domains, including toddler psychopathology, maternal stress, and parental competence. These authors also highlighted group training as feasible and acceptable to mothers, with low drop-out rates and high participation even with toddlers' involvement in sessions. In the second paper, Potharst et al. (2021b) examined a different mindful parenting training with parents of older children $\left(\mathrm{M}_{\text {age: }} 8.9\right.$ and 11.7 in the clinical and nonclinical settings, respectively). In this study, Potharst et al. evaluated the training program in both a clinical and nonclinical setting to assess its impact as a "curative" vs. preventive intervention. Notably, the authors found no significant differences in parent or child outcomes between the two groups at the 8-week follow-up. This is clinically meaningful as it emphasizes mindful parenting as an effective tool for a wide range of families, not just those with histories of psychopathology.

Two final intervention studies mark the end of the current issue. Boekhorst et al. (2021) expanded previous work by evaluating the effectiveness of an online mindful parenting program. The authors noted significant improvements across parents' use of mindful parenting practices as well as depressive and anxiety symptoms. Furthermore, their findings underscored the acceptability of mindful parenting programs to a wide range of parents and also highlighted the online format's increased accessibility and cost-effectiveness as additional advantages for parents. Telehealth interventions have been previously recommended as a potential solution to access disparities (Alegría et al. 2016). Given the diverse range of samples included in the Special Issue, Boekhorst et al.'s work and its implications for such disparities present a promising avenue for future work in this area. Lastly, Emerson et al. (2021) explored parenting predictors of improvement following participation in a mindful parenting intervention. In this respect, the authors' work provides important insight into how parent and child factors might interact and influence intervention outcomes. For example, the authors suggested that parents' engagement in mindful parenting may improve not only parents' own self-regulation skills but also their co-regulatory abilities, vicariously impacting their child's behavior. This is important as understanding these underlying mechanisms enhances our ability to personalize programs to the unique needs of each family.

While the Special Issue contributes important international perspectives to the discussion of mindful parenting, there is still much work to be done in exploring how mindful parenting presents across underrepresented populations. More specifically, the literature would benefit from an improved understanding of mindful parenting as it relates to fathers as well as racial and ethnic minorities. Still, as the current issue has emphasized, the benefits of improving mindful parenting are wide reaching. Many of the papers in this issue have examined the impact of mindful parenting in families at risk or with a history of dysfunctional parent-child interactions. In this respect, mindful parenting has not only demonstrated to be a highly acceptable component to treatment, but one with long-term benefits across multiple facets of family functioning (e.g., parent-youth communication, youth psychosocial health, parenting stress). In addition, and perhaps most important, the Special Issue spotlights recent investigations that are using prospective and randomized experimental designs, large sample sizes, and multi-method and multilevel assessment methods. Overall, these papers represent promising steps forward in the advancement of mindful parenting research, and we hope that readers find the work to be both informative and inspiring, as there is much that remains undiscovered.

\section{References}

Alegría, M., Alvarez, K., Ishikawa, R. Z., DiMarzio, K., \& McPeck, S. (2016). Removing obstacles to eliminating racial and ethnic disparities in behavioral health care. Health Affairs, 35(6), 991-999. https://doi.org/10.1377/hlthaff.2016.0029.

Anālayo, B. (2021). The Buddha and his son. Mindfulness. Advance of Print. https://doi.org/10.1007/s12671-020-01394-7.

Boekhorst, M., Hulsbosch, L. P., Nyklicek, I., Spek, V., Kastelein, A., Bogels, S. M., Pop, V. J. M., \& Potharst, E. S. (2021). An online mindful parenting training for mothers raising toddlers: assessment of acceptability, effectiveness and personal goals. Mindfulness. Advance of Print.

Bögels, S. M., Hellemans, J., van Deursen, S., Römer, M., \& van der Meulen, R. (2014). Mindful parenting in mental health care: effects on parental and child psychopathology, parental stress, parenting, coparenting, and marital functioning. Mindfulness, 5(5), 536-551. https://doi.org/10.1007/s12671-013-0209-7.

Brown, S. M., Bender, K. A., Bellamy, J. L., Garland, E. L., Dmitrieva, J., \& Jenson, J. M. (2021). A pilot randomized trial of a mindfulnessinformed intervention for child welfare-involved families. Mindfulness. Advance of Print. https://doi.org/10.1007/s12671018-1001-5.

Burgdorf, V. L., Szabo, M., \& Abbott, M. (2019). The effect of mindful interventions for parents on parenting stress and youth psychological outcomes: a systematic review and meta-analysis. Frontiers in Psychology, 10, 1336. https://doi.org/10.3389/fpsyg.2019.01336.

Calvete, E., Gómez-Odriozola, J., \& Orue, I. (2021). Differential susceptibility to the benefits of mindful parenting depending on child dispositional mindfulness. Mindfulness. Advance of Print. https://doi. org/10.1007/s12671-020-01467-7.

Casanueva, C., Tueller, S., Dolan, M., Testa, M., Smith, K., \& Day, O. (2015). Examining predictors of re-reports and recurrence of child maltreatment using two national data sources. Children and Youth Services Review, 48, 1-13. https://doi.org/10.1016/j.childyouth. 2014.10.006.

Chaplin, T. M., Turpyn, C. C., Fischer, S., Martelli, A. M., Ross, C. E., Leichtweis, R. N., et al. (2021). Parenting-focused mindfulness intervention reduces stress and improves parenting in highly stressed mothers of adolescents. Mindfulness. Advance of Print. https://doi. org/10.1007/s12671-018-1026-9.

Dumas, J. E. (2005). Mindfulness-based parent training: Strategies to lessen the grip of automaticity in families with disruptive children. 
Journal of Clinical Child and Adolescent Psychology, 34(4), 779791. https://doi.org/10.1207/s15374424jccp3404_20.

Duncan, L. G. (2007). Assessment of mindful parenting among parents of early adolescents: development and validation of the Interpersonal Mindfulness in Parenting Scale. Pennyslvania: The Pennsylvania State University.

Emerson, L. M., Aktar, E., de Bruin, E., Potharst, E., \& Bögels, S. (2021). Mindful parenting in secondary child mental health: Key parenting predictors of treatment effects. Mindfulness. Advance of Print. https://doi.org/10.1007/s12671-019-01176-w.

Fernandes, D. V., Canavarro, M. C., \& Moreira, H. (2021a). The mediating role of parenting stress in the relationship between anxious and depressive symptomatology, mothers' perception of infant temperament, and mindful parenting during the postpartum period. Mindfulness. Advance of Print. https://doi.org/10.1007/s12671020-01327-4.

Fernandes, D. V., Canavarro, M. C., \& Moreira, H. (2021b). Mindful parenting interventions for the postpartum period: acceptance and preferences of mothers with and without depressive symptoms. Mindfulness. Advance of Print. https://doi.org/10.1007/s12671020-01430-6

Han, Z. R., Ahemaitijiang, N., Yan, J., Hu, X., Parent, J., Dale, C., DiMarzio, K., \& Singh, N. N. (2021). Parent mindfulness, parenting, and child psychopathology in China. Mindfulness. Advance of Print. https://doi.org/10.1007/s12671-019-01111-z.

Hélie, S., Poirier, M. A., \& Turcotte, D. (2014). Risk of maltreatment recurrence after exiting substitute care: impact of placement characteristics. Children and Youth Services Review, 46, 257-264. https:// doi.org/10.1016/j.childyouth.2014.09.002.

Henrichs, J., van den Heuvel, M. I., Witteveen, A. B., Wilschut, J., \& Van den Bergh, B. R. (2021). Does mindful parenting mediate the association between maternal anxiety during pregnancy and child behavioral/emotional problems? Mindfulness. Advance of Print. https://doi.org/10.1007/s12671-019-01115-9.

Kabat-Zinn, M., \& Kabat-Zinn, J. (1997). Everyday blessings: the inner work of mindful parenting. New York: Hyperion.

Kabat-Zinn, J., \& Kabat-Zinn, M. (2021). Mindful parenting: perspectives on the heart of the matter. Mindfulness. Advance of Print.

Lengua, L. J., Ruberry, E. J., McEntire, C., Klein, M., \& Jones, B. (2021). Preliminary evaluation of an innovative, brief parenting program designed to promote self-regulation in parents and children. Mindfulness. Advance of Print. https://doi.org/10.1007/s12671018-1016-y.

Li, F., \& Godinet, M. T. (2014). The impact of repeated maltreatment on behavioral trajectories from early childhood to early adolescence. Children and Youth Services Review, 36, 22-29. https://doi.org/10. 1016/j.childyouth.2013.10.014.

Lippold, M. A., Jensen, T. M., Duncan, L. G., Nix, R. L., Coatsworth, J. D., \& Greenberg, M. T. (2021). Mindful parenting, parenting cognitions, and parent-youth communication: bidirectional linkages and mediational processes. Mindfulness. Advance of Print. https://doi. org/10.1007/s12671-019-01119-5.

Lönnberg, G., Jonas, W., Bränström, R., Nissen, E., \& Niemi, M. (2021). Long-term effects of a mindfulness-based childbirth and parenting program - a randomized controlled trial. Mindfulness. Advance of Print. https://doi.org/10.1007/s12671-020-01403-9.

Moreira, H., Caiado, B., \& Canavarro, M. C. (2021). Is mindful parenting a mechanism that links parents' and children's tendency to experience negative affect to overprotective and supportive behaviors? Mindfulness. Advance of Print. https://doi.org/10.1007/s12671020-01468-6.

Parent, J., Dale, C., McKee, L., \& Sullivan, A. D. (2021). The longitudinal influence of caregiver dispositional mindful attention on mindful parenting, parenting practices, and youth psychopathology. Mindfulness. Advance of Print. https://doi.org/10.1007/s12671020-01536-x.

Potharst, E. S., Baartmans, J. M., \& Bögels, S. M. (2021a). Mindful parenting training in a clinical versus non-clinical setting: an explorative study. Mindfulness. Advance of Print.

Potharst, E. S., Leyland, A., Colonnesi, C., Veringa, I. K., Jakschik, M., Bogels, S. M., \& Zeegers, M. A. J. (2021b). Does mothers' selfreported mindful parenting relate to the observed quality of parenting behavior and mother-child interaction? Mindfulness. Advance of Print.

Potharst, E. S., Zeegers, M., \& Bogels, S. M. (2021c). Mindful with your toddler group Training: Feasibility, acceptability, and effects on subjective and objective measures. Mindfulness. Advance of Print. https://doi.org/10.1007/s12671-018-1073-2.

Ren, Y., Han, Z. R., Ahemaitijiang, N., \& Zhang, G. (2021). Maternal mindfulness and school-age children's emotion regulation: mediation by positive parenting practices and moderation by maternal perceived life stress. Mindfulness. Advance of Print.

Singh, N. N., Lancioni, G. E., Medvedev, O. N., Hwang, Y. S., \& Myers, R. E. (2021). A component analysis of the mindfulness-based positive behavior support (MBPBS) program for mindful parenting by mothers of children with autism spectrum disorder. Mindfulness. Advance of Print.

Townshend, K., Jordan, Z., Stephenson, M., \& Tsey, K. (2016). The effectiveness of mindful parenting programs in promoting parents' and children's wellbeing: a systematic review. JBI Database of Systematic Reviews and Implementation Reports, 14(3), 139-180. https://doi.org/10.11124/JBISRIR-2016-2314.

Turpyn, C. C., Chaplin, T. M., Fischer, S., Thompson, J. C., Fedota, J. R., Baer, R. A., \& Martelli, A. M. (2021). Affective neural mechanisms of a parenting-focused mindfulness intervention. Mindfulness. Advance of Print. https://doi.org/10.1007/s12671-019-01118-6.

Publisher's Note Springer Nature remains neutral with regard to jurisdictional claims in published maps and institutional affiliations. 